\title{
Obesity and inflammatory markers effect on grafts blood flow in off-pump coronary artery bypass - preliminary report.
}

\author{
Tomasz Urbanowicz ${ }^{1}$, Bartłomiej Perek ${ }^{1}$, Anna Olasinska-Wisniewska ${ }^{1}$, Michal Michalak ${ }^{1}$, \\ Michal Bocianski ${ }^{1}$, Michał Rodzki ${ }^{1}$, Ewa Straburzyńska-Migaj ${ }^{1}$, and Marek Jemielity ${ }^{1}$ \\ ${ }^{1}$ Poznan University of Medical Sciences
}

July 25, 2021

\begin{abstract}
Background: Coronary artery bypass grafting is still a therapy of choice for complex ischemic heart disease. The purpose of the study was to compare the relation between obesity and blood flow through aorto-coronary bypass grafts with coexisting preoperative inflammatory state presented neutrophil-to-lymphocyte and platelets-to-lymphocyte ratios in retrospective analysis. Methods: We analyzed 50 consecutive patients (mean age $65+/-8$ ) who underwent off-pump coronary artery bypass grafting (OPCAB) in our department in 2018. Graft blood flow measurements, as well as platelet-to-lymphocyte (PLR) and neutrophilto-lymphocyte (NLR) ratios were evaluated. Results: Obese patients undergoing arterial revascularization were characterized by statistically significant lower arterial grafts flow for RIMA ( $p=0.0043)$, LIMA ( $p=0.0023)$ and RA ( $\mathrm{p}=0.0214)$ with satisfactory medium term $(897+/-123$ days) results. The chronic inflammatory parameters including NLR and PLR were significantly differed between obese and non-obese patients $(\mathrm{p}=0.0312$ and $\mathrm{p}=0.0003$, respectively) referred for surgery. The inverse correlation between BMI and NLR $(r=0.307)$ and PLR $(r=0.413)$ was noted. Conclusion: Obese patients referred for CABG present a decreased graft blood flow velocity combined with an increased neutrophil-to-lymphocyte and platelet-to-lymphocyte ratio.
\end{abstract}

Title: Obesity and inflammatory markers effect on grafts blood flow in off-pump coronary artery bypass preliminary report.

\section{Authors:}

Tomasz Urbanowicz PhD, Cardiac Surgery and Transplantology Department, Poznan University of Medical Sciences. This author takes responsibility for all aspects of the reliability and freedom from bias of the data presented and their discussed interpretation. Prof. Bartłomiej Perek, Cardiac Surgery and Transplantology Department, Poznan University of Medical Sciences. This author takes responsibility for all aspects of the reliability and freedom from bias of the data presented and their discussed interpretation. Anna OlasińskaWiśniewska PhD, Cardiac Surgery and Transplantology Department, Poznan University of Medical Sciences. This author takes responsibility for all aspects of the reliability and freedom from bias of the data presented and their discussed interpretation. Michał Michalak PhD, Department of Computer Science and Statistics, Poznan University of Medical Sciences. This author takes responsibility for all aspects of the reliability and freedom from bias of the data presented and their discussed interpretation. Michał Bociański MD, Cardiac Surgery and Transplantology Department, Poznan University of Medical Sciences. This author takes responsibility for all aspects of the reliability and freedom from bias of the data presented and their discussed interpretation. Michal Rodzki MD, Cardiac Surgery and Transplantology Department, Poznan University of Medical Sciences. This author takes responsibility for all aspects of the reliability and freedom from bias of the data presented and their discussed interpretation Prof. Ewa Straburzyńska Migaj, 1st Cardiology Department, Poznan University of Medical Sciences. This author takes responsibility for all aspects of the reliability and freedom from bias of the data presented and their discussed interpretation. Prof. Marek Jemielity. Cardiac Surgery and Transplantology Department, Poznan University of Medical 
Sciences. This author takes responsibility for all aspects of the reliability and freedom from bias of the data presented and their discussed interpretation.

Running title: Obesity vs NLR and PLR effect in OPCAB.

\section{Corresponding author:}

Tomasz Urbanowicz, MD PhD Department of Cardiac Surgery and Transplantology Poznan University of Medical Sciences, $\frac{1}{2}$ Dluga Street, 61-848 Poznan, Poland Phone No: +48-61-854-9210 (Institution) / +48605-552-551 (corresponding author) Email: tomasz.urbanowicz@skpp.edu.pl

Abstract:

Background: Coronary artery bypass grafting is still a therapy of choice for complex ischemic heart disease. The purpose of the study was to assess the relation between obesity and blood flow through aorto-coronary bypass grafts with coexisting preoperative inflammatory state presented as neutrophil-to-lymphocyte and platelets-to-lymphocyte ratios in a retrospective analysis.

Methods: We analyzed 50 consecutive patients (mean age $65+/-8$ ) who underwent off-pump coronary artery bypass grafting (OPCAB) in our department in 2018. Graft blood flow measurements, as well as platelet-to-lymphocyte (PLR) and neutrophil-to-lymphocyte (NLR) ratios were evaluated.

Results: Obese patients undergoing arterial revascularization were characterized by statistically significant lower arterial grafts flow for RIMA ( $\mathrm{p}=0.0043)$, LIMA $(\mathrm{p}=0.0023)$ and RA $(\mathrm{p}=0.0214)$ with satisfactory medium term $(897+/-123$ days) results. The chronic inflammatory parameters including NLR and PLR significantly differed between obese and non-obese patients $(\mathrm{p}=0.0312$ and $\mathrm{p}=0.0003$, respectively). The inverse correlation between BMI and NLR $(r=0.307)$ and PLR $(r=0.413)$ was noted.

Conclusion: Obese patients referred for CABG present a decreased graft blood flow velocity combined with an increased neutrophil-to-lymphocyte and platelet-to-lymphocyte ratio.

\section{Key words:}

Coronary artery disease, by-pass grafting, OPCAB, neutrophil-to-lymphocyte ratio, plateletsto-lymphocyte ratio

\section{Introduction:}

Coronary artery disease (CAD) is one of the major concerns with multifocal risk factors, including genetic and lifestyle-related [1]. Coronary artery bypass grafting is still a therapy of choice for complex ischemic heart disease and yields satisfactory long-term outcomes [2].

One of the key cardiovascular pathogenetic processes is the loss of the endothelium's protective role [3]. Its' pro-atherosclerotic phenotype is characterized by a reduced nitric oxide production compounded with vasoconstricting overactivity [4]. It has been postulated that vascular endothelial dysfunction secondary to reduced nitric oxide production, oxidative stress, and chronic inflammation is linked with obesity [5]. The relationship between severity of obesity and reduction of endothelial function affecting coronary arteries has already been proved [6.7]. Impaired vasodilatory capacity of coronary circulation precedes structural alterations and carries significant prognostic information [8].

Recently, many authors underlined the impact of mutual correlation between blood cellular components, such as platelet-to-lymphocyte (PLR) or neutrophil-to-lymphocyte (NLR) ratios, and the outcomes of both cardiac and non-cardiac medical interventions [9-11].

In this study, the predominant purpose was to estimate blood flow through aorto-coronary bypass grafts implanted on the beating heart in obese and non-obese patients and to determine its association with the results of the patients' preoperative blood morphology [12].

\section{Material and methods}




\section{Patients}

We retrospectively analyzed 50 consecutive patients (44 male and 6 females, mean age $65 \pm 8$ years) who underwent off-pump coronary artery bypass grafting (OPCAB) in our department in 2018. There were 19 $(38 \%)$ patients with a body mass index (BMI) over $30 \mathrm{~kg} / \mathrm{m}^{2}$ (obese group - OB), and 31 (62\%) with a BMI below this value (non-obese group - nOB) The mean follow-up was $897+/-123$ days.

Patients were qualified for surgery based on coronary angiography results. Twenty-nine patients (58\%) were diagnosed with the left main disease. All patients underwent arterial revascularization including left and right internal mammary artery and left radial artery. Prevalence of concomitant diseases in both groups is outlined in Table 1.

On the day of admission, blood samples for complete blood count and biochemical tests were collected. Special attention was paid to platelets and white blood cells count along with their fractions. Then, PLR and NLR were calculated for both groups. In addition, transthoracic echocardiography $(\mathrm{M}+2 \mathrm{D}+\mathrm{Doppler})$ was performed.

All patients signed written informed consent for routine surgery for CAD. Blood samples analysis and blood flow measurements were performed with standard of care. The study received positive agreement of the Local Ethics Committee.

\section{Surgical technique}

All the procedures were performed via complete median sternotomy on the beating heart, without cardiopulmonary bypass support (OPCAB). The only applied grafts were arterials including left and right internal mammary and left radial artery. Upon completion of graft harvesting, heparin in an initial dose of $2 \mathrm{mg} / \mathrm{kg}$ was administered to achieve the therapeutic range of activating clotting time (ACT) (exceeding 400s; in our group $488 \pm 37 \mathrm{~s}$ ). To facilitate distal anastomoses of the aorto-coronary bypass grafts, the deep pericardial stitch to elevate the beating heart and tissue stabilizer (Octopus III, Medtronic, USA) to immobilize segment of the recipient coronary artery, were used. All the anastomoses were performed with a continuous monofilament 7-0 suture following application of intraluminal shunts (Medtronic, USA). The size was chosen individually on the base of coronary artery diameter and wall quality to allow blood flow (and, consequently, distal myocardial perfusion) and to limit bleeding (diameters of shunts used in both groups and with respect to the target artery are presented in Table 2). After final anastomosis heparin action was reversed by two doses of protamine administered under ACT guidance to achieve its baseline value (in our group, the final ACT was $132 \pm 36$ s).

Blood flow measurements

The graft blood flow measurements were routinely performed in every surgical revascularization procedure in our department. In every case, they were performed following the second dose of protamine when patient was hemodynamically stable. Ultrasonography equipped with a $6.5 \mathrm{MHz}$ linear transducer (Verify Q, USA) was used.

Measurements were performed for left and right internal mammary arteries (LIMA and RIMA) approximately $10 \mathrm{~cm}$ from its origins from subclavian arteries, while for radial artery (RA) - 5cm from the proximal anastomosis to the ascending aorta.

Postoperative period

Following the surgery, all patients were transferred to the postoperative intensive care unit (ICU) where their vital functions were carefully monitored. Electrocardiography (ECG) was carried out immediately upon their admission to the ICU, and then on a daily basis. Serum concentrations of troponin-I were measured every 12 hours for 3 days following the surgery and maximal values were recorded for a further statistical analysis. Upon discharge, all patients were referred the outpatient clinic follow-up.

Statistical analysis 
Continuous variables were reported as mean \pm standard deviation (SD) when data followed a normal distribution; otherwise, the data were presented as medians and interquartile range $\left[\mathrm{Q}_{1}-\mathrm{Q}_{3}\right]$, where $\mathrm{Q}_{1}$ is lower and $\mathrm{Q}_{3}$ upper quartile, respectively. The differences between groups were calculated by means of unpaired Student's t-test (normally distributed) or the Mann-Whitney U test (the rest, eg. shunt diameters or troponin-I concentrations). Categorical variables were reported as numbers (n) with percentages (\%) and then compared by test for proportions. Moreover, the strengths of association between the selected blood morphology-derived ratios (PLR, NLR) and body mass index (BMI) were estimated using Pearson's parametric correlation $(\mathrm{r})$ method. All tests were considered significant at $\mathrm{p}<0.05$. The analysis was performed using Statistica 13 statistical package (TIBCO Software Inc. (2017), Statistica (data analysis software system), version 13. http://statistica.io.).

\section{Results}

Clinical outcomes

There were no perioperative deaths or myocardial infarctions in the presented groups. Median values of maximum troponin-I serum level were $1.05 \mathrm{mcg} / \mathrm{L}(0.62-6.89 \mathrm{mcg} / \mathrm{L})$ in the OB group, and $1.3 \mathrm{mcg} / \mathrm{L}(0.97-$ $3.41 \mathrm{mcg} / \mathrm{L})$ in the nOB group (ns). The mean number of performed grafts were $2,2+/-0,6(93+/-7 \%$ revascularization rate). A two-year follow-up $(897+/-123$ days) $(29.9+/-4.1$ months $)$ was completed for 48 individuals (96\%) and revealed $96 \%$ survival rate without episodes of documented myocardial infarctions.

Blood flow in arterial grafts

In the OB group, the median value of graft measurement blood flow through RIMA was $6 \mathrm{~mL} / \mathrm{min}$ (4-13) with pulsation index (PI) of $3.3 \pm 1.3$, through LIMA $-10 \mathrm{~mL} / \mathrm{min}(3-13)$ with PI of $3.1 \pm 1.2$, and through $\mathrm{RA}-2 \mathrm{~mL} / \mathrm{min}(2-11)$ with PI of $3.1 \pm 1.2$, respectively.

In the nOB group, median flow through RIMA was $15 \mathrm{~mL} / \mathrm{min}$ (8-27) with PI of 3.1 \pm 1.4 , through LIMA $18 \mathrm{~mL} / \mathrm{min}$ (9-25) with PI of $3.1 \pm 1.0$, and through RA $-16 \mathrm{~mL} / \mathrm{min}$ (14-34) with PI of $3.3 \pm 1.2$, respectively. All calculated medians of arterial grafts flow were significantly higher in the nOB group and $\mathrm{p}$ value was 0.0043 for RIMA, 0.0023 for LIMA, and 0.0214 for RA (Figure 1).

Parameters of inflammation

Preoperative whole blood count data including two commonly accepted indices of inflammatory reaction, platelet-to-lymphocyte ratio (PLR) and neutrophil-to-lymphocyte ratio (NLR), are presented in Table 1. These two parameters were significantly higher in the OB group.

\section{GRAFT FLOW and NLR}

Higher values of NLR were associated with lower blood flow. Even more interestingly, a significant $(\mathrm{p}<0.05)$ although moderate positive correlation was found between BMI and the aforementioned inflammation indices (Figures 2 and 3), and a negative one between BMI and LAD diameter $(\mathrm{r}=-0.34)$. Additionally, a significant $(\mathrm{p}<0.05)$ yet also only moderate negative correlation $(\mathrm{r}=-0.33)$ was noted between LAD diameter at the site of anastomosis and PLR.

\section{Discussion:}

To the best of our knowledge, this is the first study presenting reduced graft blood flow results in obese patients which also correlate with increased NLR and PLR. Medium 2-year results ( $897+/-123$ days) were satisfactory in obese group despite significant differences in arterial grafts flow.

According to our study, obesity was not a significant risk factor for higher perioperative mortality after coronary artery bypass grafting which is consistent with previous with previous reports [13.14]. Our results of OPCAB suggest an underestimated value of low blood flow velocity in obesity-related grafts. The mean values of arterial grafts blood flow in obese and non-obese patients were significantly different in our study with mean values of $6 \mathrm{~mL} / \mathrm{min}(4-13)$ vs $15 \mathrm{~mL} / \mathrm{min}(8-27)$ and $10 \mathrm{~mL} / \mathrm{min}(3-13)$ vs $18 \mathrm{~mL} / \mathrm{min}(9-25)$ in RIMA and LIMA, respectively. The differences in grafts' blood flow were not related to maximum values of 
postoperative Troponin-I serum levels $3.62(1.01-25.25) \mathrm{mcg} / \mathrm{L}$ vs $3.37(2.37-9.16) \mathrm{mcg} / \mathrm{L}$ in obese and non-obese patients, respectively $(\mathrm{p}=0.07414)$.

We showed statistically significant difference between blood flow measurements between overweight and nonoverweight patients. Coronary blood flow measurements can be regarded as one of the significant risk factors for cardiovascular complications in obese patients since coronary microvascular dysfunction is superior to BMI in risk prediction [15]. Coronary arteries diameter and flow velocity are two determinants of myocardial oxygen supply requirements [16]. Both the blood flow and troponin serum levels are believed to be strong indicators of future major adverse cardiovascular events [17-19]. There was no case of periprocedural myocardial infarction in our study group. We revealed a reduced graft flow in obese patients which was not related to periprocedural ischemia/injury.

During off-pump surgery, the intraluminal shunts are used to facilitate performance of anastomosis. The non-significant difference in diameters of shunts used allow to conclude that the results obtained in graft blood flow measurement are not related to diameters or wall quality of coronary arteries. The mean values of shunts applied during anastomoses presented in Table 2 confirm the hypothesis that not a coronary artery diameter is responsible for diminished blood flow but chronic inflammatory processes presented by NLR and PLR. Obese patients presented higher NLR with mean values of $3.5+/-1.3$ vs $2.7+/-1(\mathrm{p}=0.0312)$. The PLR results, second indirect marker of inflammation, were also significantly different between obese and normal weight patients with mean values of $221+/-81$ vs $142+/-60(\mathrm{p}=0.0003)$, respectively. The unique nature of the inflammatory response to obesity was already postulated sharing some similarities with other chronic inflammatory processes. This trigger provoked by energy homeostasis disruption over time leads to maladaptive response [20].

Osadnik et al compared NLR and PLR results as significant factors of chronic inflammation between obese and non-obese patients [21]. The increased NLR and PLR were characteristic for obese patients in present study. The reduced blood flow reserve in chronic inflammatory states have already been observed [22.23].

Our study results present the blood flow significant differences in coronary grafts between obese and nonobese patients. Analysis based on patients who underwent arterial revascularization as possible best option with satisfactory long-term graft patency rates though recent studies indicate more further studies [24]. The significant difference noted can be explained with a correlation between BMI and increased inflammatory parameters as a sign of inflammatory state. BMI results were correlated with PLR and NLR ratios. There was as inverse correlation observed between PLR (chronic inflammatory marker) and mean diameters of the anastomosed coronary artery.

Previous reports suggested that the increased level of serum CRP (C-reactive protein) was a possible trigger for intimal hyperplasia and risk for calcification in grafts applied during coronary artery bypass grafting procedures [24]. Subclinical atherosclerosis estimated by intimal thickening of arteries is related to serum adiponectin levels in obese patients [25]. These reports are presenting a possible explanation of our results and confirm our hypothesis of chronic inflammation as a causative agent. In a multicenter analysis, Schwann confirmed the correlation between higher mortality and morbid obesity in early and long-term periods and a partial protective role of "the obesity paradox" in the early and intermediate postoperative periods in overweight and mildly obese patients [26]. The poorer long-term results following coronary artery bypass grafting (increased 5-year and 10-year mortality rates) in obese patients were also observed despite the immediate good survival rates [27].

There is a considerable body of evidence suggesting that endothelial signals modulate the blood flow [27] and may therefore be responsible for the discrepancy between obese and non-obese patients presented in our results.

Study limitation is related to retrospective analysis of small sample group who underwent arterial coronary artery bypass grafting performed as single center study related to midterm results.

\section{Conclusion:}


Obese patients referred for CABG present a decreased blood flow velocity combined with an increased NLR and PLR. Our findings should warrant further research regarding the impact of systemic and local inflammation on the coronary artery flow.

Author contributions:

Tomasz Urbanowicz - I, III, IV, V, VI, VIIBartłomiej Perek - I, IV, V, VI, VIIAnna OlasińskaWisniewska - III, IV, V, VI, VIIMichał Michalak - I, V, VI, VIIMichał Rodzki - III, VI, VIIMichał Bociański - III, VI, VIIEwa Straburzyńska-Migaj - V, VI, VIIMarek Jemielity - II, V, VI, VII.

\section{References:}

1. AK Malakar, D Choudhury, B Halder, P Paul, Uddin A, Chakraborty S. "A review on coronary artery disease, its risk factors, and therapeutics." J Cell Physiol.2019;234(10):16812-16823.

2. Modolo R, Chichareon P, Kogame N, Dressler O, Crowley A, Ben-Yehuda O, Puskas J, Banning A, Taggart DP, Kappetein AP, Sabik JA, Onuma Y, Stone GW, Serruys PW. "Contemporary Outcomes Following Coronary Artery Bypass Graft Surgery for Left Main Disease." J Am Coll Cardiol.2019;73(15):1877-1886.

3. A Virdis, R Colucci, N Bernardini, C Blandizzi, S Taddei, S Masi. "Microvascular Endothelial Dysfunction in Human Obesity: Role of TNF-a." J Clin Endocrinol Metab.2019;104(2):341-348.

4. VirdisA, TaddeiS. "Endothelial dysfunction in resistance arteries of hypertensive humans: old and new conspirators." J Cardiovasc Pharmacol.2016;67(6):451-457.

5. A Virdis, S Masi, R Colucci, M Chiriaco, M Uliana, I Puxeddu, N Berdardini, C Blandizzi, S Taddei. "Microvascular Endothelial Dysfunction in Patients with Obesity." Curr Hypertens Rep.2019;21(4):32-37.

6. F Perticone, R Ceravolo, M Candigliota, G Ventura, S Iacopino, F Sinopoli, PL Mattioli. "Obesity and body fat distribution induce endothelial dysfunction by oxidative stress: protective effect of vitamin C." Diabetes. 2001;50(1):159-165.

7. J Al Suwaidi, ST Higano, DR Holmes Jr, R Lennon, A Lerman. "Obesity is independently associated with coronary endothelial dysfunction in patients with normal or mildly diseased coronary arteries." J Am Coll Cardiol.2001;37(6):1523-1528.

8. TH Schindler, HR Schelbert, A Quercioli, V Dilsizian. "Cardiac PET imaging for the detection and monitoring of coronary artery disease and microvascular health." J Am Coll Cardiol Img.2010;3(6):623-40.

9. H Li, Y Zhou, Y Ma, S Han, L Zhou. "The prognostic value of the platelet-to-lymphocyte ratin acute coronary syndrome: a systematic review and meta-analysis." Kardiol Pol.2017;75(7):666-673.

10. E Bressi, F Mangiacapra, E Ricottini, I Cavallari, I Colaiori, G Di Gioia, A Creta, M Capuano, MM Viscusi, GJ Di Sciascio. "Impact of neutrophil-to-lymphocyte ratio and platelet-to-lymphocyte ratio on 5 -year clinical outcomes of patients with stable coronary artery disease undergoing elective percutaneous coronary interventions." Cardiovasc Transl Res.2018;11(6):517-523.

11. J Yu, Z Ding, Y Yang, S Liu. "Increased platelet-to-lymphocytes ration is associated with poor long-term prognosis in patients with pancreatic cancer after surgery." Medicine (Baltimore).2018;97(25):e11002.

12. Taggart DP, Thuijs DJFM, Di Giammarco G, Puskas JD, Wendt D, Trachiotis GD, Kieser TM, Kappetein AP, Head SJ. Intraoperative transit-time flow measurement and high-frequency ultrasound assessment in coronary artery bypass grafting. J Thorac Cardiovasc Surg. 2020 Apr;159(4):1283-1292.e2.

13. R Jin, GL Grunkemeier, AP Furnary, JR Handy Jr. Providence Health System Cardiovascular Study Group. "Is Obesity a Risk Factor for Mortality in Coronary Artery Bypass Surgery?" Circulation.2005;111(25):3359-3365. 
14. AHM van Straten, S Bramer, M Hamad, AJ van Zundert, EJ Martens, J Schönberger, AM de Wolf. "Effect of Body Mass Index on Early and LateMortality After Coronary Artery Bypass Grafting." Ann Thorac Surg.2010:89(1):30-37.

15. NS Bajaj, MT Osborne, A Gupta, A Tavakkoli, P E Bravo, T Vita, CF Bibbo, J Hainer, S Dorbala, R Blanckstein, DL Bhatt, MF Di Carli VR Taqueti. "Coronary microvascular dysfunction and cardiovascular risk in obese patients." J Am Coll Cardiol.2018;72(7):707-717.

16. PA van Diemen, SP Schumacher, MJ Bom, RS Driessen, H Everaars, WJ Stuijfzand, PG Raijmakers, PM van de Ven, JK Min, JA Leipsic, J Knuuti, PR Boellaard, CA Taylor, AC van Rossum, P Knaapen. "The association of coronary lumen volume to left ventricle mass ratio with myocardial blood flow and fractional flow reserve." J Cardiovasc Comput Tomogr.2019;13(4):179-187.

17. W Bedaux, M Hofman, S Vyt, J Bronzwaer, C Visser, A van Rossum. "Assessment of Coronary Artery Bypass Graft Disease Using Cardiovascular Magnetic Resonance Determination of Flow Reserve." J Am Coll Cardiol.2002;40(10):1848-1855.

18. E Mauermann, D Bolliger, J Fassl, M Grapow, EE Seeberger, MD Seeberger, M Filipovic, GAL Lurati Buse. "Postoperative High-Sensitivity Troponin and Its Association With 30-Day and 12-Month, All-Cause Mortality in Patients Undergoing On-Pump Cardiac Surgery." Anesth Analg. 2017;125(4):1110-1117.

19. P Markman, JP Tantiongco, JS Bennetts, R Baker. "High-Sensitivity Troponin Release Profile After Cardiac Surgery." Heart Lung Circ.2017;26(8):833-839.

20. Saltiel AR, Olefsky JM. "Inflammatory mechanisms linking obesity and metabolic disease." J Clin Invest.2017;127(1):1-4.

21. T Osadnik, K Bujak, K Osadnik, H Czarnecka, N Pawlas, R Reguła, M Fronczek, M Lejawa, M Gawlita, M Gonera, M Góral, JK Strzelczyk, M Gierlotka, A Lekston, J Kasperczyk, L Poloński, M Gąsior. "Novel inflammatory biomarkers may reflect subclinical inflammation in young healthy adults with obesity." Endokrynol Pol.2019;70(2):135-142.

22. E Pesonen, $\mathrm{P}$ Liuba, EH Aburawi. "Review findings included diminished coronary flow reserve after surgery in children with congenital heart disease and inflammation." Acta Paediatr.2019;108(2):218-223.

23. J Li, H Zhang, C Zhang. "Role of inflammation in the regulation of coronary blood flow in ischemia and reperfusion: mechanisms and therapeutic implications." J Mol Cell Cardiol. 2012;52(4):865-872.

24. Sef D, Raja SG. Bilateral internal thoracic artery use in coronary artery bypass grafting in the post-ART era - Perspective. Int J Surg. 2021 Feb;86:1-4.

25. S De Marchi, E Falleti, R Giacomello, G Stel, E Cecchin, G Sepiacci, N Bortolotti, F Zanello, F Gonano, E Bartoli. "Risk factors for vascular disease and arteriovenous fistula dysfunction in hemodialysis patients." J Am Soc Nephrol.1996;7(8):1169-1177.

26. M Shargorodsky, M Boaz, Y Goldberg, Z Matas, D Gavish, A Fux, N Wolfson. "Adiponectin and vascular properties in obese patients: is it a novel biomarker of early atherosclerosis?" Int J Obes,2009;33(5):553-538.

27. TS Schwann, PS Ramia, MC Engoren, MR Bonnell, M Goodwin, I Monroe, HR Habib. "Evidence and temporality of the obesity paradox in coronary bypass surgery: an analysis of cause-specific mortality." Eur J Cardiothorac Surg.2017;54(5):896-903.

28. T Terada, M Forhan, CM Norris, W Qiu, R Padwal, AM Sharma, J Nagendran, JA Johnson. "Differences in Short- and Long-Term Mortality Associated with BMI Following Coronary Revascularization." J Am Heart Assoc.2017;6(4):e005335.

29. XB Meng, Q Fu, WY Sun, JM Yu, WM Yue, YW Bi. "Competitive flow arising from varying degrees of coronary artery stenosis affects the blood flow and the production of nitric oxide and endothelin in the internal mammary artery graft." Euro Journ of Cardio-Thoracic Surg.2013;43(5):1022-1027. 


\section{Table 1.}

Demographic and clinical data of the entire study group $(n=50)$.

Abbreviations: ACS - acute coronary syndrome, BMI - body mass index, COPD - chronic obstructive pulmonary disease, DM - diabetes mellitus, NLR - neutrophil-to-lymphocyte ratio, (n)OB - (non-)obese patients, PLT - platelet-to-lymphocyte ratio, WBC - white blood cells.

Table 2. Intraoperative blood flow measurements and additional intra- and postoperative data.

Abbrevations:

$\mathrm{Cx}$ - circumflex artery, hrs - hours, LAD - left anterior descending artery; LIMA - left internal mammary artery; LRA - left radial artery, LVEF - left ventricular ejection fraction, (n)OB - (non-)obese patients, RCA - right coronary artery, RIMA - right internal mammary artery.

Figure 1.

Blood flow differences in implanted grafts between obese and normal weight patients.

Abbreviations:

LIMA - left internal mammary artery, Min - minimum, Max- Maximum, RIMA - right internal mammary artery.

\section{Figure 2.}

Correlation between BMI vs NLR.

Abbreviations:

BMI - body mass index, NLR - neutrophil to lymphocyte ratio

Figure 3.

Correlation BMI vs PLR.

Abbreviations:

BMI - body mass index, PLR - platelet to lymphocyte ratio.

Hosted file

Obesity Table 1.docx available at https://authorea.com/users/337664/articles/531613-obesityand-inflammatory-markers-effect-on-grafts-blood-flow-in-off-pump-coronary-artery-bypasspreliminary-report

\section{Hosted file}

Obesity Table 2.docx available at https://authorea.com/users/337664/articles/531613-obesityand-inflammatory-markers-effect-on-grafts-blood-flow-in-off-pump-coronary-artery-bypasspreliminary-report 

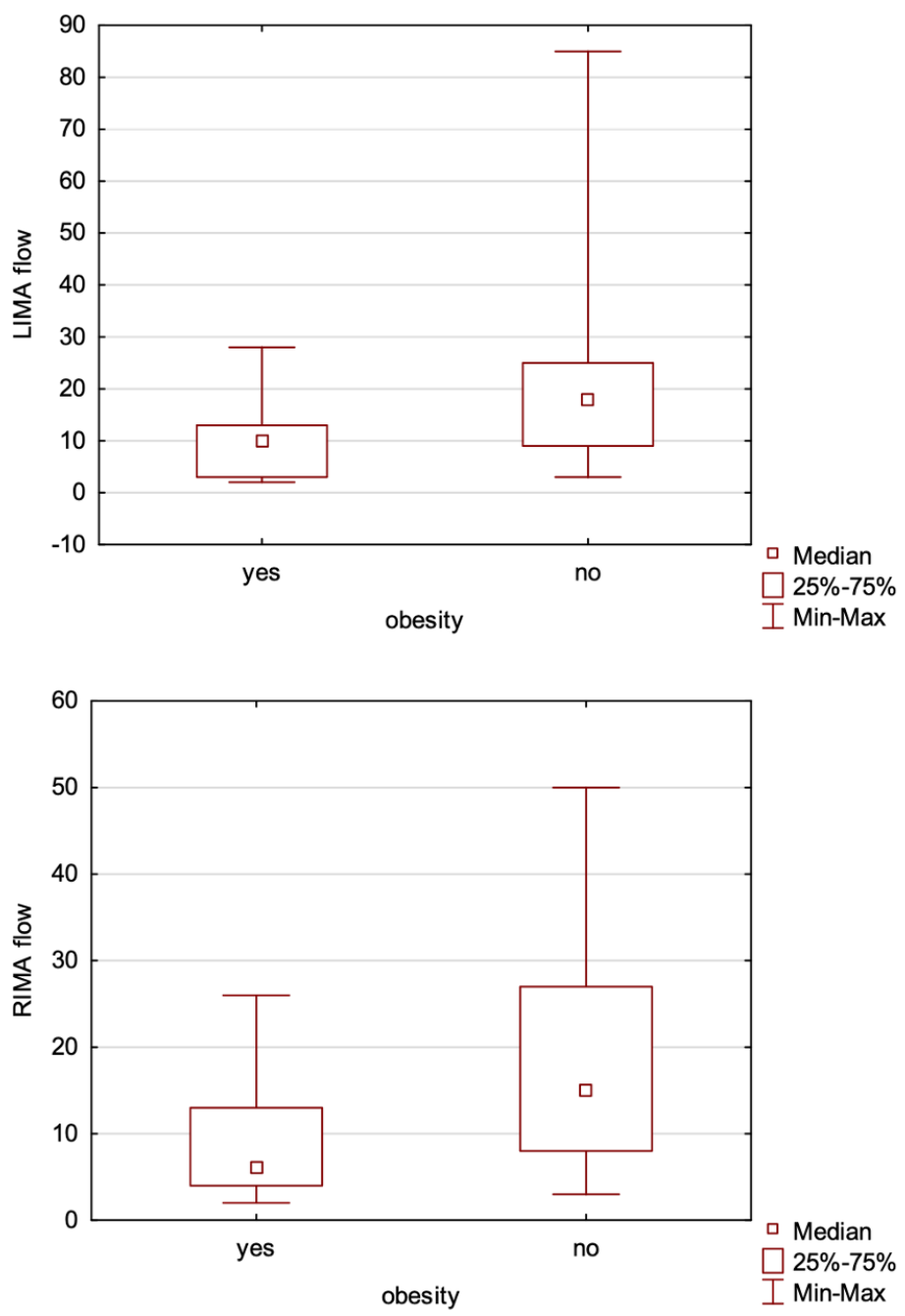

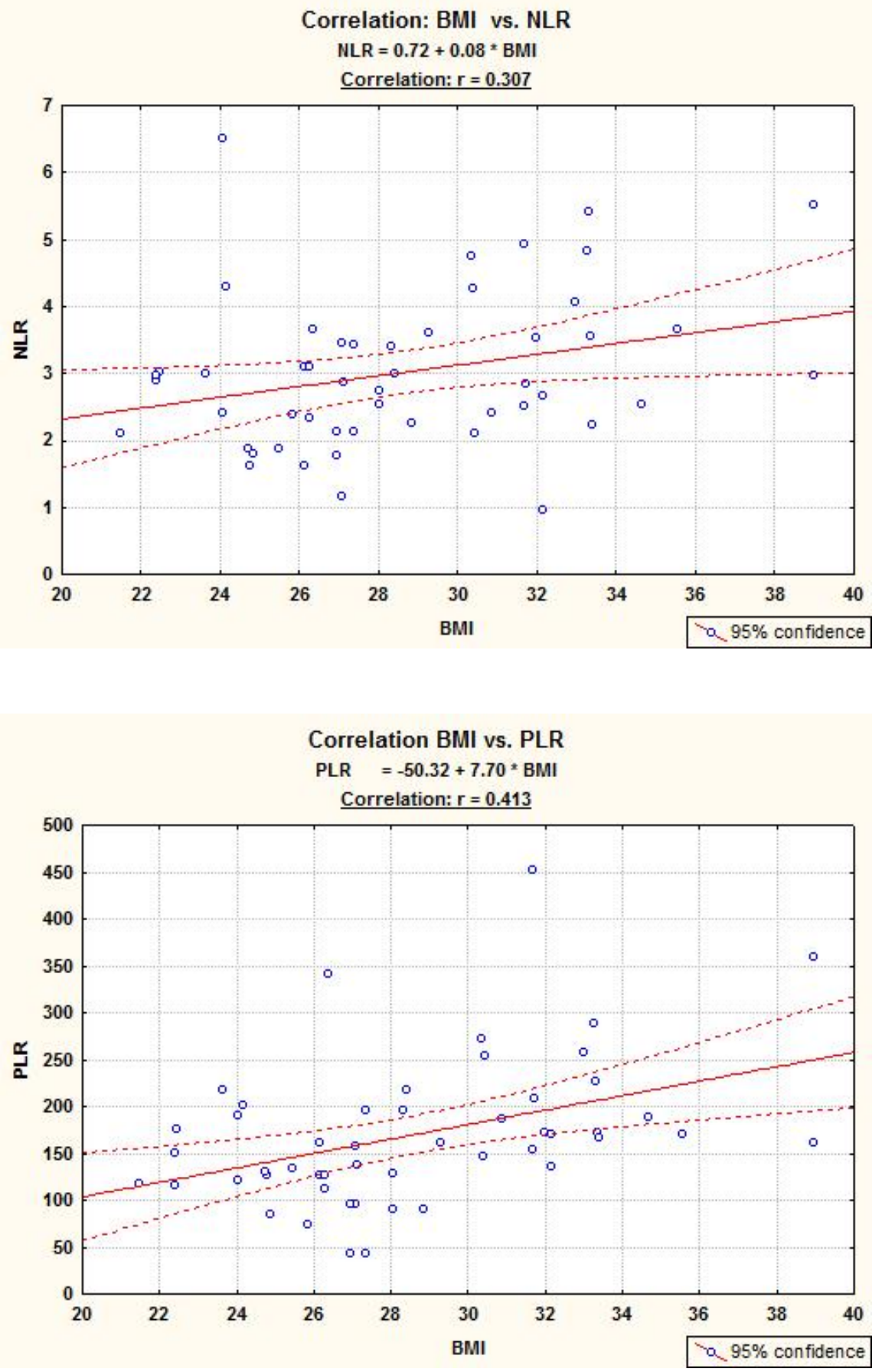